\title{
Dynamic response of two-dimensional electron liquids in the local-density-functional theory
}

\author{
B. Tanatar \\ Department of Physics, Bilkent University, Bilkent, 06533 Ankara, Turkey
}

(Received 3 January 1991)

\begin{abstract}
The exchange-correlation potentials, $f_{\mathrm{xc}}(q=0, \omega)$, to be used in the local-density approximation to the density-functional theory for two-dimensional homogeneous electron liquids in the normal and fully spin-polarized phases are calculated. We make use of accurate Monte Carlo energies and sum rules in the local-field correction, $G(q, \omega)$, to construct $f_{\mathrm{xc}}(q, \omega)$ which is valid in the long-wavelength limit. Our results are qualitatively similar to the three-dimensional case.
\end{abstract}

\section{INTRODUCTION}

The density-functional theory proposed by Hohenberg and Kohn ${ }^{1}$ and Kohn and Sham ${ }^{2}$ provides an excellent tool to study the ground-state properties of condensedmatter systems. The success of local-density approximation (LDA) in time-independent problems is well known, so it is very important to develop and test its counterpart for time-dependent ones, especially in connection with response theory. Recently, Gross and Kohn ${ }^{3}$ extended the density-functional formalism to treat the dynamic (linear) response of electron liquids within the LDA. They constructed an exchange-correlation functional that satisfied some exact conditions, including the compressibility and third-frequency moment $\left(\left\langle\omega^{3}\right\rangle\right)$ sum rules. In their application of the formalism to three-dimensional (3D) electron liquids, using an approximate form for the $\left\langle\omega^{3}\right\rangle$ sum rule, correlation effects were partially neglected. Iwamoto and Gross ${ }^{4}$ corrected the condition based on the $\left\langle\omega^{3}\right\rangle$ sum rule and obtained a strong frequency dependence of the exchange-correlation functional even though it was evaluated in the long-wavelength limit. A detailed review of the subject of time-dependent densityfunctional theory and its applications is recently given by Gross and Kohn. ${ }^{5}$

In the method of Gross and Kohn, ${ }^{3}$ contact is made with the homogeneous electron gas, of which the theoretical formulation is based on the dynamic local-field correction $G(k, \omega)$. There have been several attempts to construct a dynamic $G(q, \omega)$ for the elecron gas in various approximations. ${ }^{6}$ In relation to the time-dependent LDA, Dabrowski ${ }^{7}$ proposed a simple parametrized form for $G(q, \omega)$ of a $3 \mathrm{D}$ electron liquid, which satisfies the low- and high-frequency limits exactly, and generalizes the Gross and Kohn result ${ }^{3}$ to finite- $q$ values. In their study of the high-frequency damping of collective excitations in fermion systems, Holas and Singwi ${ }^{8}$ also proposed a model local-field correction $G(q, \omega)$, and formally constructed the exchange-correlation potential $f_{\mathrm{xc}}(q, \omega)$ for a two-dimensional (2D) electron liquid. At the time, the static structure factor $S(k)$ and a parametrized form of the correlation energy $\varepsilon_{c}\left(r_{s}\right)$ from Monte Carlo (MC) simulations were not available to construct $G(q, \omega)$ explicitly.
The purpose of this article is to provide explicit expressions for the exchange-correlation potentials $f_{\mathrm{xc}}(q=0, \omega)$ that enter the local-density approximation for $2 \mathrm{D}$ electron liquids both in the normal and fully spin-polarized phases. We use the results of a recent $\mathrm{MC}$ calculation ${ }^{9}$ of $2 \mathrm{D}$ electron gas to construct the model local-field correction $G(q, \omega)$ proposed by Holas and Singwi ${ }^{8}$ that satisfies the sum rules. Note that we are interested in the dynamic correlations in the density fluctuations of the electron liquid both in the normal (paramagnetic) and fully spin-polarized (ferromagnetic) cases. In particular, we do not investigate the dynamic response of the system due to spin fluctuations. The dynamic spin susceptibility within the framework of the time-dependent spin densityfunctional theory has recently been studied by Liu and Vosko. ${ }^{10}$ In the rest of this article, we first discuss the frequency dependence of the exchange-correlation potential, $f_{\mathrm{xc}}(q, \omega)$, within the linear-response density-functional theory. Our results for $f_{\mathrm{xc}}(q, \omega)$ in the normal and fully spin-polarized cases are presented in Sec. III, and we conclude with a brief summary.

\section{DYNAMIC RESPONSE IN THE DENSITY-FUNCTIONAL THEORY}

To make this note self-contained we outline the basic steps of density-functional formalism (following closely the original work of Gross and $\mathrm{Kohn}^{3}$ ) for a 2D electron liquid, and later explicitly construct the exchangecorrelation potentials required in the LDA. In the density-functional theory, ${ }^{3}$ one considers an unperturbed homogeneous electronic system with density $n_{0}(\mathbf{r})$ in the ground state of the static external potential $v_{0}(\mathbf{r})$. For a small perturbing potential $v_{1}(\mathbf{r}, t)$ and corresponding density $n_{1}(\mathbf{r}, t)$, the associated Fourier components $v_{1}(\mathbf{r}, \omega)$ and $n_{1}(\mathbf{r}, \omega)$ are related by

$$
n_{1}(\mathbf{r}, \omega)=\int d^{2} r^{\prime} \chi\left(\left|\mathbf{r}-\mathbf{r}^{\prime}\right| ; \omega\right) v_{1}\left(\mathbf{r}^{\prime}, \omega\right),
$$

where $\chi\left(\left|\mathbf{r}-\mathbf{r}^{\prime}\right| ; \omega\right)$ is the exact density-density response function. If we now assume that the density $n_{0}(\mathbf{r})+$ $n_{1}(\mathbf{r}, t)$ can be reproduced by the noninteracting system in an appropriate potential $v_{0}^{\text {eff }}(\mathbf{r})+v_{1}^{\text {eff }}(\mathbf{r}, t)$, we can write 


$$
n_{1}(\mathbf{r}, \omega)=\int d^{2} r^{\prime} \chi_{0}\left(\left|\mathbf{r}-\mathbf{r}^{\prime}\right| ; \omega\right) v_{1}^{\mathrm{eff}}\left(\mathbf{r}^{\prime}, \omega\right),
$$

where $\chi_{0}$ denotes dynamic response of a noninteracting system, i.e., the Lindhard function. Defining the exchange-correlation part of $v_{1}^{\text {eff }}$ by

$v_{1}^{\mathrm{eff}}(\mathbf{r}, \omega)=v_{1}(\mathbf{r}, \omega)+\int d^{2} r^{\prime} \frac{n_{1}\left(\mathbf{r}^{\prime}, \omega\right)}{\left|\mathbf{r}-\mathbf{r}^{\prime}\right|}+v_{1, \mathrm{xc}}(\mathbf{r}, \omega)$,

we introduce the functional relation

$$
v_{1, \mathrm{xc}}(\mathbf{r}, \omega)=\int d^{2} r^{\prime} f_{\mathrm{xc}}\left(\left|\mathbf{r}-\mathbf{r}^{\prime}\right| ; \omega\right) n_{1}\left(\mathbf{r}^{\prime}, \omega\right),
$$

where the exchange-correlation potential $f_{\mathrm{xc}}$ depends on the unperturbed ground-state density $n_{0}(\mathbf{r})$. From the above definition of $f_{\mathrm{xc}}$, we can formally write (in Fourier space),

$$
f_{\mathrm{xc}}(q, \omega)=\chi_{0}^{-1}(q, \omega)-\chi^{-1}(q, \omega)-v(q),
$$

where $v(q)$ is the Coulomb potential in $2 \mathrm{D}$, and $\chi_{0}(q, \omega)$ is the dynamic susceptibility of the noninteracting system (Lindhard function). To obtain an approximation for $v_{1, \mathrm{xc}}$, the LDA is invoked, $n_{0}(\mathbf{r})$ and $n_{1}(\mathbf{r}, \omega)$ are assumed to be slowly varying so that $f_{\mathrm{xc}}$ is evaluated for the local density $n_{0}(\mathbf{r})$, and $n_{1}\left(\mathbf{r}^{\prime}, \omega\right)$ in Eq. (4) is replaced by $n_{1}(\mathbf{r}, \omega)$. This yields

$$
v_{1, \mathrm{xc}}(\mathbf{r}, \omega) \approx f_{\mathrm{xc}}\left(q=0, \omega ; n_{0}(\mathbf{r})\right) n_{1}(\mathbf{r}, \omega) .
$$

Hence we need to find an expression for the exchangecorrelation potential $f_{\mathrm{xc}}$ of the homogeneous electron liquid in the long-wavelength limit $(q \rightarrow 0)$. Using the defining equation for the local-field correction $G(q, \omega)$

$$
\chi(q, \omega)=\frac{\chi_{0}(q, \omega)}{1+v(q) G(q, \omega) \chi_{0}(q, \omega)},
$$

we can make contact with the exchange-correlation potential

$$
f_{\mathrm{xc}}(q, \omega)=-v(q) G(q, \omega),
$$

where the Coulomb potential reads $v(q)=4 \pi / q$ (in a.u.). The above relation holds for homogeneous systems within the linear-response theory.

Holas and Singwi ${ }^{8}$ derived an asymptotic expression for the imaginary part of the dielectric function, $\operatorname{Im} \epsilon(q, \omega)$, considering a selected set of diagrams (particle-hole diagrams) in the second-order perturbation theory. They obtained the corresponding high-frequency limit for the local-field correction $G(q, \omega)$ in 2D (Ref. 8) [we use atomic units (a.u.), so that energies are in Rydbergs and lengths are in units of Bohr radius $a_{B}$ ],

$$
\lim _{\omega \rightarrow \infty} \operatorname{Im} G(q, \omega)=\frac{11 \pi}{32} \frac{q}{\omega} .
$$

Strictly speaking the above expression is only valid at high densities, i.e., $r_{s} \rightarrow 0$, but it is assumed here as in the $3 \mathrm{D}$ case $^{3}$ to hold for a wide range of densities. Then a simple form (following the work of Gross and $\mathrm{Kohn}^{3}$ ) for
$G(q, \omega)$ is proposed that interpolates between the small- $\omega$ and large- $\omega$ behaviors,

$$
G(q, \omega)=G(q, \infty)+\frac{i \omega_{1}(q)}{\omega+i \omega_{2}(q)},
$$

where $\omega_{1}(q)$ and $\omega_{2}(q)$ are $q$-dependent functions to be determined. They are obtained by taking certain limits of $G(q, \omega)$, viz.,

$$
\begin{aligned}
& \lim _{\omega \rightarrow \infty}[\omega \operatorname{Im} G(q, \omega)]=\omega_{1}(q), \\
& G(q, 0)=G(q, \infty)+\frac{\omega_{1}(q)}{\omega_{2}(q)} .
\end{aligned}
$$

Hence $\omega_{1}(q)$ and $\omega_{2}(q)$ are given by

$$
\begin{aligned}
& \omega_{1}(q)=\frac{11 \pi}{32} q, \\
& \omega_{2}(q)=\frac{\omega_{1}(q)}{G(q, 0)-G(q, \infty)} .
\end{aligned}
$$

The high- and low-frequency limits of the local-field correction $G(q, \omega)$ at long wavelengths were calculated by Iwamoto ${ }^{11}$ consistent with the compressibility and $\left\langle\omega^{3}\right\rangle$ sum rules,

$\lim _{q \rightarrow 0} G(q, \infty)=\left[\frac{5}{6 \pi} \alpha r_{s}+\frac{7}{8} \alpha^{2} r_{s}^{2} \varepsilon_{c}+\frac{19}{16} \alpha^{2} r_{s}^{3} \frac{d \varepsilon_{c}}{d r_{s}}\right] q$,

$\lim _{q \rightarrow 0} G(q, 0)=\left[\frac{\alpha r_{s}}{\pi}+\frac{\alpha^{2} r_{s}^{3}}{8} \frac{d \varepsilon_{c}}{d r_{s}}-\frac{\alpha^{2} r_{s}^{4}}{8} \frac{d^{2} \varepsilon_{c}}{d r_{s}^{2}}\right] q$

where $\alpha=2^{-1 / 2}$ and $\varepsilon_{c}$ is the correlation energy. If we further define $f_{\infty}=-\lim _{q \rightarrow 0} v(q) G(q, \infty)$ and $f_{0}=$ $-\lim _{q \rightarrow 0} v(q) G(q, 0)$, we can express the function $w_{2}(q)$ as

$$
w_{2}(q)=\frac{\left(11 \pi^{2} / 8\right)}{f_{\infty}-f_{0}} .
$$

It is clear that the exchange-correlation potential constructed above obeys all the conditions and has properties set out by Gross and $\mathrm{Kohn}^{3}$ for the 3D case, and in particular $f_{\mathrm{xc}}$ satisfies the Kramers-Kronig relations, viz.,

$\operatorname{Re} f_{\mathrm{xc}}(q, \omega)=f_{\infty}+\mathcal{P} \int_{-\infty}^{\infty} \frac{d \omega^{\prime}}{\pi} \frac{\operatorname{Im} f_{\mathrm{xc}}\left(q, \omega^{\prime}\right)}{\omega^{\prime}-\omega}$

$\operatorname{Im} f_{\mathrm{xc}}(q, \omega)=-\mathcal{P} \int_{-\infty}^{\infty} \frac{d \omega^{\prime}}{\pi} \frac{\operatorname{Re}\left[f_{\mathrm{xc}}\left(q, \omega^{\prime}\right)\right]-f_{\infty}}{\omega^{\prime}-\omega}$,

where $\mathcal{P}$ denotes the principal part of the integral. We have also checked the relation $f_{0}<f_{\infty}<0$ (for all $r_{s}$ ) for the MC data and observed that it is fulfilled for both the normal and the fully spin-polarized liquid phases.

\section{RESULTS}

We present our results of the frequency-dependent exchange-correlation potential in the long-wavelength 


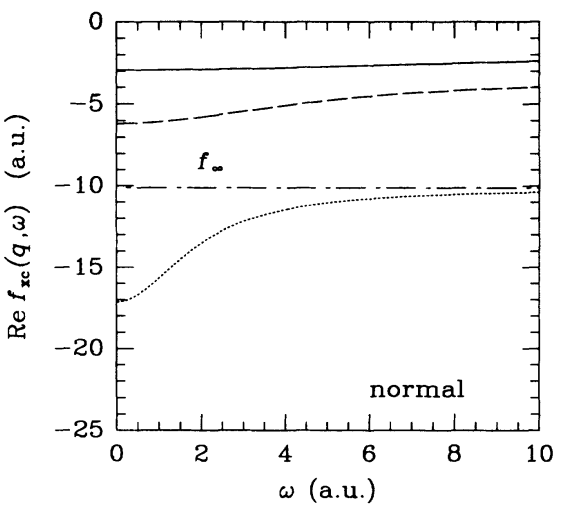

FIG. 1. Frequency dependence of the real part of the model exchange-correlation potential $f_{\mathrm{xc}}(q=0, \omega)$ for a $2 \mathrm{D}$ electron system in the normal fluid phase for $r_{s}=1$ (solid line), 2 (dashed line), and 5 (dotted line).

limit, $f_{\mathrm{xc}}(q=0, \omega)$, in the following. We display in Fig. 1 the frequency dependence of the real part of $f_{\mathrm{xc}}(q=0, \omega)$ for $r_{s}=1,2$, and 5 , indicated by solid, dashed, and dotted lines, respectively. Also shown is $f_{\infty}\left(r_{s}=5\right)$ by the dotted-dashed line. We may estimate the error that would ensue in the adiabatic approximation, when $f(q=0, \omega)$ is replaced by its value at $\omega=0$, i.e., by $f_{0}$. For $r_{s}=5$ the maximum error $\Delta=\left(f_{0}-f_{\infty}\right) / f_{0}$, would be $\sim 70 \%$. In practice, there exists a characteristic frequency of the system $0<\omega^{*}<\infty$ for which $f_{\mathrm{xc}}$ is dominant and the errors of using $f_{\mathrm{xc}}\left(\omega^{*}\right)$ rather than the full frequency range is considerably less than our estimate $\Delta$. In Fig. 2 we show the frequency dependence of the imaginary part of $f_{\mathrm{xc}}(q=0, \omega)$ for $r_{s}=1,2$, and 5 indicated by solid, dashed, and dotted lines, respectively. The behavior of $f_{\mathrm{xc}}$ is qualitatively similar to the $3 \mathrm{D}$ case, but since $\operatorname{Im} f_{\mathrm{xc}} \sim 1 / \omega$ as $\omega \rightarrow \infty$, it approaches zero slower than its counterpart in 3D.

The formalism we have set out to construct the dynamic local-field correction and the exchange-correlation

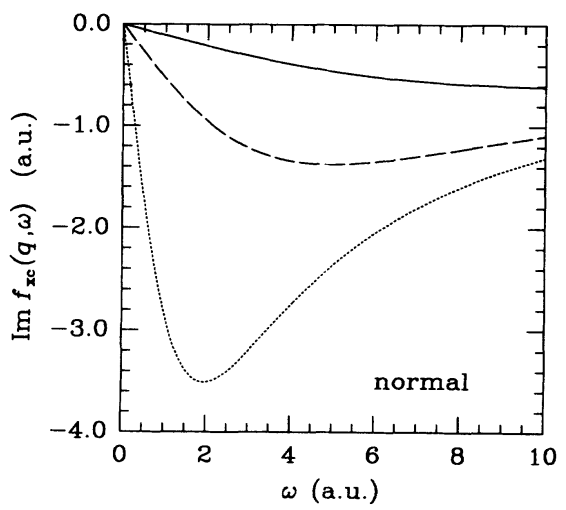

FIG. 2. Frequency dependence of the imaginary part of the model exchange-correlation potential $f_{\mathrm{xc}}(q=0, \omega)$ for a $2 \mathrm{D}$ electron system in the normal fluid phase for $r_{s}=1$ (solid line), 2 (dashed line), and 5 (dotted line).

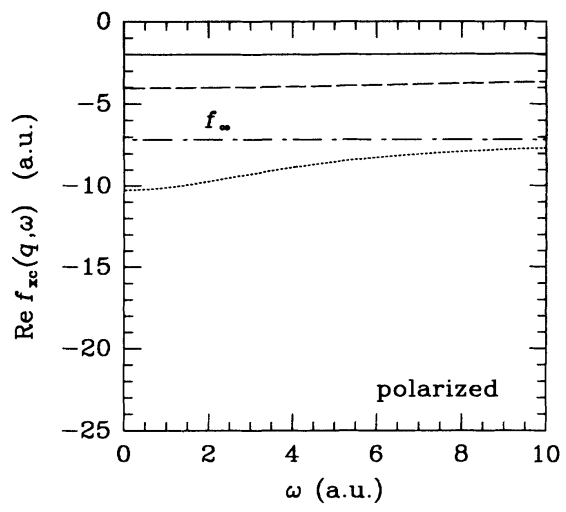

FIG. 3. Frequency dependence of the real part of the model exchange-correlation potential $f_{\mathrm{xc}}(q=0, \omega)$ for a $2 \mathrm{D}$ electron system in the spin-polarized fluid phase for $r_{s}=1$ (solid line), 2 (dashed line), and 5 (dotted line).

potential may readily be extended to calculate these quantities for the fully spin-polarized electron liquid. The correlation energy $\varepsilon_{c}\left(r_{s}\right)$ (which enters $f_{0}$ and $f_{\infty}$ ) for the spin-polarized system obtained from a MC calculation has a parametrized form. ${ }^{9}$ Note that we are not evaluating the spin-density response functions, hence the exchange-correlation potential is still given by Eq. (5) but the Fermi wave vector $q_{F}$ that enters $\chi(q, \omega)$ is the one appropriate for the fully spin-polarized liquid. Since the Fermi wave vectors of a spin-polarized and normal liquids (in 2D) are related by $q_{F}^{\uparrow}=\sqrt{2} q_{F}$, the expressions for the local-field factor $G(q, \omega)$ given in Eqs. (15) and (16) are accordingly scaled. In Figs. 3 and 4 we show the real and imaginary parts of the exchange-correlation potential $f_{\mathrm{xc}}(q=0, \omega)$ for the fully spin-polarized electron liquid. We have plotted Figs. 3 and 4 on the same scale as Figs. 1 and 2, for easy comparison. We observe that $\operatorname{Re} f_{\mathrm{xc}}$ has a somewhat stronger frequency dependence in the normal fluid phase than that in the spin-polarized case. The same is true for $\operatorname{Im} f_{\mathrm{xc}}$ and the difference in the

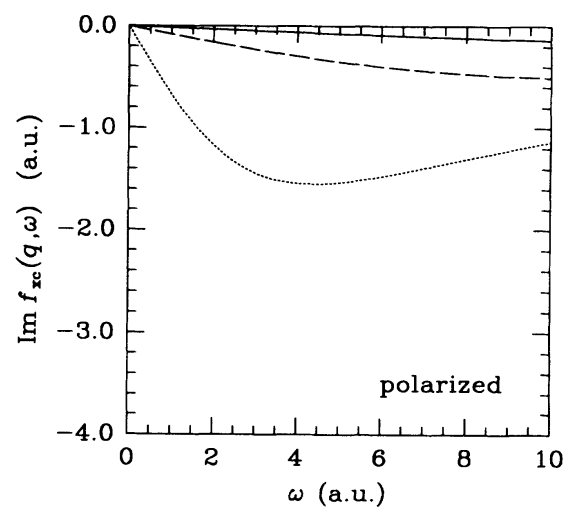

FIG. 4. Frequency dependence of the imaginary part of the model exchange-correlation potential $f_{\mathrm{xc}}(q=0, \omega)$ for a $2 D$ electron system in the spin-polarized fluid phase for $r_{s}=1$ (solid line), 2 (dashed line), and 5 (dotted line). 
high-frequency behavior of $\operatorname{Im} f_{\mathrm{xc}}$ for normal and spinpolarized electron liquids diminishes with increasing $r_{s}$ (see Figs. 2 and 4).

\section{SUMMARY}

In summary, we have presented explicit expressions for the exchange-correlation potentials $f_{\mathrm{xc}}(q=0, \omega)$ for $2 \mathrm{D}$ electron liquids in normal and spin-polarized phases as proposed by Holas and $\mathrm{Singwi}^{8}$ and the results are qualitatively similar to the $3 \mathrm{D}$ case. They should be useful in future applications of the density-functional theory to 2D homogeneous electron liquids. The time-dependent density-functional theory in $3 \mathrm{D}$ has been successfully applied to a range of problems including the photoresponse of atoms and molecules, metallic and semiconductor surfaces, and bulk semiconductors. ${ }^{12}$ The exchangecorrelation potentials obtained here could be used in similar problems where the physical system has a $2 \mathrm{D}$ charac- ter, and to the extent the linear-response theory is applicable such as the calculation of atomic polarizabilities. In this work we presented a model $f_{\mathrm{xc}}(q=0, \omega)$ similar to the $3 \mathrm{D}$ case ${ }^{3,4,8}$ using appropriate limiting forms of $G(q, \omega)$. It would be interesting to construct a $G(q, \omega)$ with the MC structure factor $S(q)$ as input or some other scheme, such as the self-consistent field approximation of Singwi et $a l^{13}$ or the approach advanced by Dabrowski, ${ }^{7}$ thereby avoiding the $q \rightarrow 0$ approximation.

\section{ACKNOWLEDGMENTS}

We would like to thank Professor Abdus Salam, the International Atomic Energy Agency and the United Nations Educational, Scientific and Cultural Organizations for their hospitality during the author's stay at the International Centre for Theoretical Physics in Trieste where most of this work was done. We gratefully acknowledge Dr. G. Pastore for his valuable suggestions.
${ }^{1}$ P. Hohenberg and W. Kohn, Phys. Rev. 136, B864 (1964).

${ }^{2}$ W. Kohn and L. J. Sham, Phys. Rev. 140, A1133 (1965).

${ }^{3}$ E. K. U. Gross and W. Kohn, Phys. Rev. Lett. 55, 2850 (1985); 57, 923(E) (1986).

${ }^{4}$ N. Iwamoto and E. K. U. Gross, Phys. Rev. B 35, 3003 (1987).

${ }^{5}$ E. K. U. Gross and W. Kohn, in Advances in Quantum Chemistry (Academic, New York, 1990), Vol. 21.

${ }^{6}$ S. Ichimaru, H. Iyetomi, and S. Tanaka, Phys. Rep. 149, 91 (1987); S. Ichimaru, Rev. Mod. Phys. 54, 1017 (1982), and references cited therein.

${ }^{7}$ B. Dabrowski, Phys. Rev. B 34, 4989 (1989).
${ }^{8}$ A. Holas and K. S. Singwi, Phys. Rev. B 40, 158 (1989).

${ }^{9}$ B. Tanatar and D. M. Ceperley, Phys. Rev. B 39, 5005 (1989).

${ }^{10}$ K. L. Liu and S. H. Vosko, Can. J. Phys. 67, 1015 (1989).

${ }^{11}$ N. Iwamoto, Phys. Rev. A 30, 3289 (1984). We noticed a typographical error in this paper in Eq. (4.18a). In the second term (the term proportional to $\alpha r_{s} \varepsilon_{c}$ ) the numerator should read $\left(d^{2}-1\right)(4 d-1)$ instead of $4(d-1)^{2}(d+1)$.

${ }^{12}$ See Ref. 5 for a complete discussion of the applications of time-dependent density-functional theory.

${ }^{13}$ K. S. Singwi, M. P. Tosi, R. H. Land, and A. Sjölander, Phys. Rev. 176, 589 (1968). 